\title{
Self-mode-locking of a semiconductor laser using positive feedback
}

\author{
D. J. Derickson, F. J. Helkey, A. Mar, P. A. Morton, and J. E. Bowers \\ Department of Electrical and Computer Engineering, University of California, Santa Barbara, \\ Californin 93106
}

(Received 23 August 1989; accepted for pubication 30 October 1989)

\begin{abstract}
A new mode-locking technque, self-mode-locking, is described which uses the detected optical pulses from the mode-locked laser as the active driving scurce. This technique forms narrowwidth mode-locked optical puises at low repetition rates without the use of a microwave synthesizer.
\end{abstract}

Active-mode locking of semiconductor lasers has produced optical pulses as short as $0.58 \mathrm{ps}$. 'This result was achieved using the positive portion of a $16 \mathrm{GHz}$ sinusoid as an electrical drive signal. For many applications, such as electro-optic sampling, it is more useful to have the modelocked optical pulses at a much lower repetition rate. This requires an electrical drive source that produces short electrical pulses at this lower rate. Step recovery diodes can be used, but they typically have puise widths greater than 50 ps. Since the mode-locked optical pulses themselves are short, they are good candidates to drive the mode-locking action. This letter describes a new mode-locking technique called self-mode-locking that uses a high-speed optical to electrical $(O / E)$ converter in a positive feedback configuration to convert the output optical pulses back into electrical drive signals in a regenerative process. Previous works ${ }^{2,3}$ have used positive feedback in a self-gain-switching configuration. Self-mode-locking is different in that both coordinated optical and electrical feedback are involved and much shorter pulses can be obtained. Advances in high-speed electrical components allow the short optical pulse to generate a short electrical drive pulse. Thotodetectors have been shown to be capable of producing impulse responses of less than $10 \mathrm{ps}^{4}$ Since the photocurrent is ton small to directly drive the laser, an amplifier is necessary to boost the feedback signal. Broadband distributed amplifers have produced $3 \mathrm{~dB}$ bandwidths of over $30 \mathrm{GHz}^{5}$ and new device technologies promise to extend this bandwidth even further. Consequently, this technique can produce shor mode-locked pulses at lower repetition rates, and it can do this without the reed for an externa electrical drive source.

The block diagram of the self-mode-locked semiconductor laser is shown in Fig. 1. A semiconductor laser is placed in an external ring cavity configuration with a round trip delay time of $2 \mathrm{~ns}$. The laser is a high-speed $1300 \mathrm{~nm}$ semiinsulating planar buried hererostructure laser ${ }^{6}$ with antireflection coatings on both facets. The light from the laser is collimated by the use of two antireffection-coated graded index lenses. The $O / E$ converter ${ }^{7}$ consists of a high-speed $p-i-n$ photodetector and a four-stage metal-semiconductor field-effect transistor distributed amplifer. The $O / E$ converter has an overall responsivity of $20 \mathrm{a} / \mathrm{W}$. Impulse response measurements of the $O / E$ converter using $1060 \mathrm{rm}, 3$ ps optical pulses show that it is capable of producing $50 \mathrm{ps}$ full width at half maximum (FWHM) pulses with a peak ampirtude of $3.5 \mathrm{~V}$ into a $50 \Omega$ system.
The self-mode-locking action occurs as follows. The laser is first biased above the cw lasing threshold. Counterpropagating optical signals start to build up in the ring cavity. One of the two output signas is fed into an $O / E$ converter. This optical signal is then converted into an electrical drive signal and apphed to the direct moduiation input of the laser. If the round trip delay of the optical cavity and the round trip delay of the signal that goes through the $O / E$ converter are properly chosen, a regenerative process will build up a mode. locked pulse in the laser. In order for self-mode-locking to occur, the loop gain of the $O / E$ converter feedback path, $R \eta h v / q$, must be greater than $1 . R$ is the $O / E$ converter responsivity, $\eta$ is the laser differential quantum efficiency, $h$ is Plarck's constant, $v$ is the optical frequency, and $q$ is the electronic charge. The other condition necessary for selfoscillation is that the optical path time delay $T_{o}$ must be related to the electrical path time delay $T_{e}$ by the following expression:

$$
T_{0} / n=T_{e} / m \text {. }
$$

This condition forces the electrical pulses and the optical pulses to arrive in the gain section of the laser at the same ime. For stable operation, the electrical delay time must be shortened slightly to allow the carrier density to rise before the arrival of the optical pulse. Figure 2 illustrates this timing relationship for the case of $n=2$ and $m=3$. The electrical path delay is 5 times the optical path delay. The optical cavity has two sets of counterpropagating pulses and the electrical cavity has three propagating pulses. The time between the successive pulses in both loops is nearly identi-



FIG. 1. Experimental setup for sclf-mode-locking. Therc are two feedback loops: one for the optical signel, and one for the optical to electrical converter. 


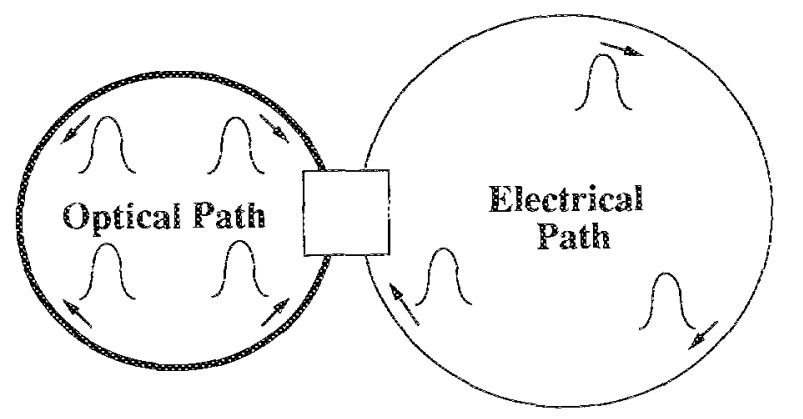

FIG. 2. Circulating electrical and optical puises in the lasef for the case where the electrical path delay is 1.5 times the optical path delay $(n \ldots 2$, $m=3$ ).

cal. The pulse repetition frequency of the self-mode-locked laser is $f=n / T_{0}$. Figure 3 shows the measured average power versus de current drive of the self-mode-locked laser for the case of $n=3$ and $m=10$. Curve $A$ in the figure shows the response without $O / E$ converter feedback. When feedback is added (curve $B$ ), the laser breaks into pulsation just above the cw lasing threshold. For this particular system, the laser amplitude limits when the peak feedback current available from the $O / E$ converter is reached. Curve $B$ shows that the average power of the self-mode-locked laser increases with laser bias but with a lower diferential quantum efficiency than the cw value. Curve $C$ shows that once self-mode-locking has been established, the laser can be biased below the cw lasing threshold and it will continue to oscillate. The hysteresis shown is possible because the $O / E$ converter suppiics the extra current necessary to maintain the oscillation below de threshold.

Using the system of Fig. 1, pulse repetition frequencies from $500 \mathrm{MHz}(n=1, m=3)$ to $6 \mathrm{GHz}(n=12, m=37)$ have been measured. The pulse rate is adjusted by substituting in various lengths of coaxial transmission line delays and changing the length of the optical ring cavity for fine adjustments. 1 has been found experimentally that if two solutions of Eq. (1) result in nearly the same cavity lengths, the mode with the lowest value of $n$ actually occurs. Figure 4 shows a plot of the second-harmonic intensity autocorrelation of a

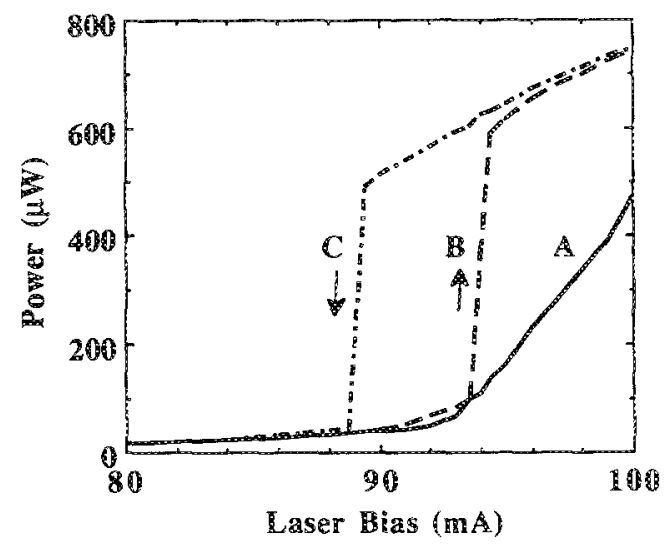

FIG. 3. Right output vs current for the self-mode-locked laser. Trace A shows the measurement withoui optical to electrical converter fectback. Fraces $B$ and $C$ show the measurement with $O / E$ converter feedback.

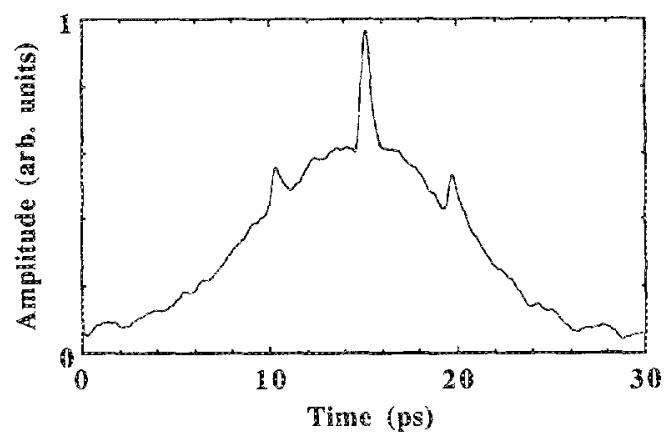

TG. 4. Sccond-harmonic trace of a $1.5 \mathrm{GHz}(n-3, m=10)$ repetition rate output pulse. The optical pulse FWHM is 8 ps assuming a hyperbolic secant squared pulse shape.

$1.5 \mathrm{GHz}(n=3, m=10)$ pulse train. If a hyperbolic secant squared pulse shape is assumed, the autocorrelation gives an optical pulse FWHM of 8 ps.

The width of the optical pulse is very sensitive to the relative lengths of the optical and electrical feedback loops. Figure 5 shows a plot of the optical pulse FWHM versus changes in the optical path deiay with the electrical path delay held constant for a $1.5 \mathrm{GHz}$ ( $n=3, n=10$ ) case. The left-hand side of the plot shows the condition of the electrical pulse arriving at the laser 30 ps earlier than the optical pulse. By shortening the optical cavity (or by lengthening the electrical cavity), the pulse width narrows until the optical puise starts to arrive too late at the laser to achieve significant optical gain and the optical pulse width rises dramatically.

Figure 5 also shows the theoretical detuning behavior which agrees qualitatively with the experimental data. The theoretical results are based on a partial integration solution of the traveling-wave rate equations. "The modeled current drive for the system has a de bias plus a component due to the $O / E$ feedback. This $O / E$ feedback current is digitally filtered and limited to match the measured impulse response and saturation characteristics of the $O / E$ converter.

In summary, this letter describes a new lype of mode locking in which an external oscillator is not needed. This self-mode-locking process uses the output of the laser itself to drive the mode-locking action. The system can be made to



FIG. 5. Optical pulse width vs arrival time difference between the electrical and optical pulses at the gain region. 
pulse at harmonics of the fundamental cavity frequency by proper choice of feedback delays. The pulse repetition rate of the system can be made lower than the drive rates usually used for short pulse generation is actively mode-locked semiconducter lasers. Self-mode-locking would also work with hybrid passive and active node-locked lasers. 'The saturable absorber curent could be used to drive the feedback amplifier directly, eliminating the need for an extemal photodetector.

This work was supported by the Offce of Naval Research. The authors acknowledge useful discussions with Mark Rodwell and Rory Van Tuyl.
'S. Corzine, J. Bowers, B. Miler, G. Przbylek, U. Koren, B. Miner, and C. Socolich, Appl. Phys. Lett. 52, 348 (1988).

2T. Damen and M. Duguay, Electron. Lett. 16, 166 (1980).

${ }^{3}$ K. Lau and A. Yariv, Appl. Phys. Lett. 45, 124 (1984).

4J. Howers and C. Burrus Jr, J. Lightwave Technol. LT-5, 1339 (1987).

${ }^{5} \mathrm{~J}$. Orr, IEEE MTT-S Inter. Microwave Symp. 10, 817 (1986).

"J. Eowers, U. Koren, B. I. Miller, C. Soccolich, and W. Y. Jan, Electron. Leit. 23, 1263 ( 1987$).$

7D. Derickson, C. Miller, and R. Van Tuyl, IEEE MTT-S Inter, Microwave Symp. 12, 1063 (1988).

${ }^{8}$ R. J. Helkey, P. A. Morton, and J. E. Bowers, Opt. Lett. 15, 15 Jan (1990). "F. A. Morton, J. E. Bowers, L. A. Koszi, M. Soler, J. Eopata, and D. F. Wilt, Appl. Phys. Lett. 56, 8 Jan. (1990). 